\title{
Putative candidate genes responsible for leaf rolling in rye (Secale cereale L.)
}

\author{
Beata Myśków ${ }^{1 *}$ D, Magdalena Góralska ${ }^{1}$, Natalia Lenarczykk ${ }^{1}$,lona Czyczyło-Mysza ${ }^{2}$ and Stefan Stojałowski ${ }^{1}$
}

\begin{abstract}
Background: Rolling of leaves (RL) is a phenomenon commonly found in grasses. Morphology of the leaf is an important agronomic trait in field crops especially in rice; therefore, majority of the rice breeders are interested in RL. There are only few studies with respect to RL of wheat and barley; however, the information regarding the genetic base of RL with respect to the shape of leaf in rye is lacking. To the best of our knowledge, this is the first study on the localization of loci controlling RL on high density consensus genetic map of rye.

Results: Genotypic analysis led to the identification of 43 quantitative trait loci (QTLs) for RL, grouped into 28 intervals, which confirms the multigenic base of the trait stated for wheat and rice. Four stable QTLs were located on chromosomes 3R, 5R, and 7R.

Co-localization of QTL for RL and for different morphological, biochemical and physiological traits may suggests pleiotropic effects of some QTLs. QTLs for RL were associated with QTLs for such morphological traits as: grain number and weight, spike number per plant, compactness of spike, and plant height. Two QTLs for RL were found to coincide with QTLs for drought tolerance (4R, 7R), two with QTLs for heading earliness (2R, 7R), one with a-amylase activity QTL (7R) and three for pre-harvest sprouting QTL (1R, 4R, 7R).

The set of molecular markers strongly linked to RL was selected, and the putative candidate genes controlling the process of RL were identified. Twelve QTLs are considered as linked to candidate genes on the base of DArT sequences alignment, which is a new information for rye.

Conclusions: Our results expand the knowledge about the network of QTLs for different morphological, biochemical and physiological traits and can be a starting point to studies on particular genes controlling RL and other important agronomic traits (yield, earliness, pre-harvest sprouting, reaction to water deficit) and to appoint markers useful in marker assisted selection (MAS). A better knowledge of the rye genome and genes could both facilitate rye improvement itself and increase the efficiency of utilizing rye genes in wheat breeding.
\end{abstract}

Keywords: Bulliform cells, COV, FEl1 (LRR receptor-like serine/threonine-protein kinase), Jasmonate O-methyltransferase, QTL, Subtilisin-like protease, TLP (tubby-like proteins), Transcription factor bHLH (basic helix-loop-helix protein)

\section{Background}

Leaf rolling (RL) is a typical response of a plant during water deficit that is observed in various field crops such as rice, maize, wheat, and sorghum. It decreases transpiration by decreasing the effective leaf area, and thus is a potentially useful drought tolerance mechanism in dry areas [1]. Although RL is a phenomenon commonly found in grasses, it has attracted much attention from

\footnotetext{
* Correspondence: bmyskow@zut.edu.pl

${ }^{1}$ Department of Plant Genetics, Breeding and Biotechnology,

West-Pomeranian University of Technology, Szczecin, ul. Słowackiego 17,

71-434 Szczecin, Poland

Full list of author information is available at the end of the article
}

rice researchers and breeders [2]. Only a few studies reported on RL of wheat [3-5] and barley [6], but studies regarding RL of rye are lacking.

Flag RL function is an important drought tolerance mechanism, enabling the plant to conserve water by decreasing transpiration during water stress and reduce leaf temperature [4]. Some Mediterranean grasses decrease transpiration as much as 46 to $63 \%$ by RL. In many species, RL does not occur until the water content in the leaf decreases to lethal levels (Parker 1968, after [7]).

In rice, RL is classified as abaxial leaf roll (both sides of the leaf roll inward along the vein) and adaxial leaf roll (both sides of the leaf roll outward along the vein) 
according to the direction of RL [8]. Analysis of some of the leaf development mutants of Arabidopsis thaliana and maize has shown that some mutations with respect to RL are related to the development of the leaf along the adaxial-abaxial axis. Establishment of leaf polarity and cell differentiation affecting leaf shape are controlled by both transcription factors and small RNAs [2, 9]. In higher plants, two types of cells are involved in RL: bulliform and hypodermis cells. Bulliform cells, which are located in the upper epidermis of the leaf near the midrib or vascular bundles of leaves, cause rolling in some Gramineae species such as rice, maize, wheat, and Sorghum spp. [1].

Due to its importance, many studies have been performed to characterize the genes controlling RL in rice. To date, no less than 17 rice mutants with rolled leaves have been characterized [10], no fewer than 70 genes/ QTLs for the rolled leaf trait have been mapped or cloned throughout the rice genome [11], and at least 28 differentially expressed proteins related to $\mathrm{RL}$ traits have been isolated and identified [12]. A study on durum $\times$ wild emmer wheat recombinant inbred line population has reported 11 significant QTLs associated with flag RL mechanism [4]. In this study, we aimed to localize the loci controlling RL on high density consensus genetic map of rye. We also aimed to detect QTL co-localization of RL and other agronomic traits.

\section{Methods}

\section{Plant material and genetic map}

In this study, we used a population of recombinant inbred lines (RILs) namely, RIL-M, which is a cross between S120 and S76 lines. S120 and S76 were developed within the commercial breeding programs conducted at Danko Plant Breeding Ltd. (Choryń, Poland) and are partially related but are genetically different [13] with respect to the following: $\alpha$-amylase activity (AA), preharvest sprouting (PHS), heading and flowering time (HE), and different morphological traits.

Mapping population consisted of 143 genotypes of RIL- $\mathrm{F}_{8}$ generation. Consensus genetic map for the RIL-M (with consideration of the data from four rye populations) was created using the Multipoint Consensus 2.2 software package [14]. Detailed information on RIL-M mapping population and algorithms used to release genetic map are provided by Milczarski et al. [15].

All seven linkage groups accounted for 1318 markers (1256 DArTs, i.e. markers detected by Diversity Array Technology and 62 PCR-based loci). Individual chromosomes included from 117 (5R) to 257 (6R) loci and spanned the distance of $128 \mathrm{cM}$ (5R)-251 cM (7R). The whole length of map was $1355 \mathrm{cM}$, and average distance varied from $0.7 \mathrm{cM}$ to $1.9 \mathrm{cM}$.

\section{Phenotype analyses}

All the experiments in this study were conducted at West Pomeranian University of Technology, Szczecin $\left(53.45^{\circ} \mathrm{N}, 14.53^{\circ} \mathrm{E}\right)$. The RIL-M population (S7-S12 generations) consisted of 143 genotypes and were analyzed during six vegetation seasons (years: 2008, 2009, 2010, 2012, 2013, and 2017). In the years 2008-2010, genotypes of mapping population were planted and analyzed in duplicates. Each RIL was represented by 7-8 plants grown in the field or in pots under natural or near natural conditions.

Due to inbreeding depression of numerous lines, individuals representing each genotype were first germinated in a glasshouse (15th-25th September) and then (1st20th October) vital seedlings were planted manually in the field-each line into one row. Finally, 7-8 individuals were grown in each row (length of rows was $100 \mathrm{~cm}$, dimension between rows was $17.5 \mathrm{~cm}$ ). The order of lines grown in the field was random and different in each year of study for the following replicates: RL08-1, RL09-1, RL09-2, RL10-1, RL10-2, RL12, and RL17. Variants RL09-1 and RL09-2 as well as RL10-1 and RL10-2 were grown in different plots.

Genotypes of two variants (replicates RL08-2 and RL13) were grown in the buckets of $10 \mathrm{dm}^{3}$ volume filled with an equal mass $(1440 \mathrm{~g})$ of soil and sand mixture $(1: 1 \mathrm{v} / \mathrm{v})$, seven plants of each genotype together in one bucket. Seeds were sown in January; initially plants were grown at a temperature of $15{ }^{\circ} \mathrm{C}$ for first 3 weeks, followed by gradually decreasing the temperature until it approached atmospheric conditions. Further vegetation proceeded under natural conditions of the winter-summer period (February-August), with natural daylight duration.

Our plant material was characterized by adaxial rolling leaves. A visual score of the degree of RL was made on the whole plants at the tillering stage, using a 5-point scale: $1-$ no signs of rolling, 1.5 -the first evidence of rolling, 2-slightly rolled leaves, 2.5-strongly rolled leaves, and 3-completely rolled leaves (a closed cylinder). Figure 1 shows the parental lines with extreme rate of traits. The assessment was performed after rainfall or watering the plants in buckets, to avoid the effect of leaves rolling under the influence of water deficit.

\section{Statistical analysis}

QTL mapping was performed using the method of composite interval mapping (CIM) with Windows QTL Cartographer 2.5 software [8]. The step size chosen for all QTLs was $2 \mathrm{cM}$. Significant thresholds to declare the presence of a QTL were estimated from 1000 permutations of the data at $P<0.05$. In addition, nonparametric Fisher's test was conducted to point markers significantly connected with rolling leaves segregations. 

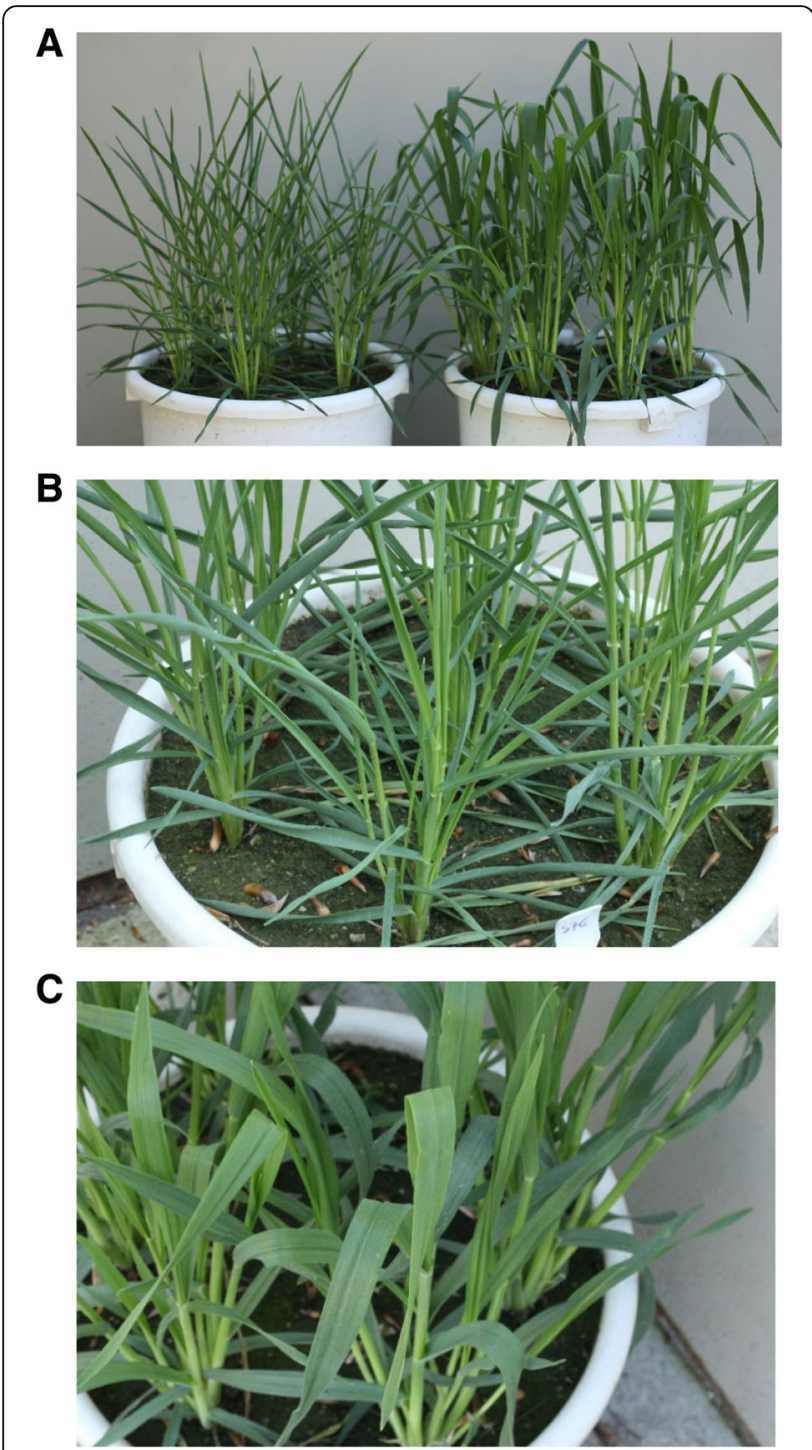

Fig. 1 Parental inbred lines of rye mapping population RIL-M, differing in terms of rolling leaves. a Paternal line S76 with rolled leaves, maternal line S120 with straight leaves, (b) line S76, and (c) line $\mathrm{S} 120$

Variation parameters and correlation coefficient between replicates was established with STATISTICA 12.0 software package (Stat-Soft, Inc., USA, http://www.statsoft.com).

Results of CIM were compared with previous QTL analyses for AA, PHS, HE [16], with recalculated data for consensus map), morphological traits [17], spike compactness, and drought index of morphological traits [18] for the same genetic map of RIL-M population.

\section{Results}

\section{Phenotypic variation and correlation analyses}

The mapping population RIL-M was characterized by an average coefficient of variation of 32.15-44.51 in terms of
$\mathrm{RL}$, depending on the replication (Table 1). Mean values of the trait ranged from 1.74 to 2.11 .

Significant positive correlations were found between results of the most pairs of replicates, and there was no correlation found in four cases (Additional file 1). Assessment of two variants in the same vegetative season showed a correlation coefficient of $0.60,0.35$, and 0.48 in the year 2008, 2009, and 2010, respectively. The weakest correlation (0.19) was noted between RL08-1 and RL09-2 and the strongest (0.60) correlation was found between RL08-2 and three other replications: RL08-1, RL10, RL17.

\section{QTL analyses}

Putative QTLs for RL were detected in each season except the year 2013. A total of 43 QTLs grouped in 28 intervals on all 7 chromosomes (2-8 per chromosome) were identified (Fig. 2). There were seven groups of coinciding QTLs found on chromosomes $3 R, 4 R, 5 R$, and $7 R$; four of them were detected 3-5 times (Fig. 2, Table 2).

The QTLs detected were found to be responsible for $6.61-21.00 \%$ of the variation in the trait. Eighteen loci were characterized with coefficient of variation more than 10\%. LOD values ranged from 2.09 to 7.34 . Twenty-two QTLs peaks achieved LOD value above 3.0. The highest LOD was achieved by locus from 5RS. Absolute value of parameter $a$ ranged from 0.21 to 0.51 . Most of the alleles causing RL $(31 ; 16$ if consider common localization) were contributed by S76; however, 12 alleles responsible for RL originated from S120.

Thirty-five markers were indicated as the nearest to the QTL peaks: 31 DArTs, 2 random amplified polymorphic DNAs (RAPDs), and 2 sequence characterized amplified regions (SCARs); among them, three loci were revealed twice (XrPt507717, XrPt507812, and XrPt400276), and two loci were revealed three times (XrPt507473 and Xpr665L1050).

Figure 2 shows the location of all QTLs. If any locus was found in different replicates, then we presented those only as a single gray rectangle.

In addition to CIM, the nonparametric, Fisher's $(F)$ test was used to detect markers significantly linked to the phenotype of rolled leaves. A total of 155 loci from 6 chromosomes were pointed as linked to the trait at least once, most of them (46) from chromosome 7R. Chromosome $1 \mathrm{R}$ had no representation; $2 \mathrm{R}$ was represented by one marker. By limiting the pool of markers to those detected at least thrice, a set of 128 loci was obtained (Table 3). Only 15 out of 35 loci nearest to the QTL peaks were in this group. Two strongly linked markers from $7 \mathrm{R}$ were revealed by $F$ test in all 9 replications of the experiment. The other 26 markers were detected 8 times; among them, 1 marker from 5R, 3 from 3R, and the rest from $7 R$ (Table 3). Next, 26 loci were found to 
Table 1 Parameters of phenotypic variation and correlation coefficient ( $r$ ) of leaf rolling (RL) in rye mapping population RIL-M, assessed in nine experimental replications

\begin{tabular}{|c|c|c|c|c|c|c|c|c|c|c|}
\hline \multirow{2}{*}{$\begin{array}{l}\text { Experiment } \\
\text { replication }\end{array}$} & \multirow{2}{*}{$\begin{array}{l}\text { Number of } \\
\text { genotypes } \\
\text { evaluated } N\end{array}$} & \multirow{2}{*}{$\begin{array}{l}\text { RL parameter } \\
A \vee\end{array}$} & \multirow[t]{2}{*}{ Variance } & \multirow[t]{2}{*}{ SD } & \multirow{2}{*}{$\begin{array}{l}\text { Coefficient of } \\
\text { variation }\end{array}$} & \multirow[t]{2}{*}{ SE } & \multirow[t]{2}{*}{ Skewness } & \multirow[t]{2}{*}{ Kurtosis } & \multicolumn{2}{|l|}{ r } \\
\hline & & & & & & & & & Min & Max \\
\hline RL08-1 & 139 & 1.92 & 0.71 & 0.84 & 43.90 & 0.07 & 0.15 & -1.58 & $0.36^{*}$ & $0.60^{*}$ \\
\hline RL08-2 & 90 & 1.76 & 0.59 & 0.77 & 43.80 & 0.08 & 0.45 & -1.17 & 0.25 & $0.60^{*}$ \\
\hline RL09-1 & 142 & 1.83 & 0.61 & 0.78 & 42.64 & 0.07 & 0.31 & -1.30 & $0.29^{*}$ & $0.47^{*}$ \\
\hline RL09-2 & 140 & 1.74 & 0.51 & 0.71 & 40.93 & 0.06 & 0.42 & -0.95 & 0.19 & $0.40^{*}$ \\
\hline RL10-1 & 138 & 1.82 & 0.60 & 0.78 & 42.66 & 0.07 & 0.33 & -1.27 & 0.26 & $0.60^{*}$ \\
\hline RL10-2 & 131 & 1.75 & 0.61 & 0.78 & 44.51 & 0.07 & 0.47 & -1.20 & 0.25 & $0.48^{*}$ \\
\hline RL12 & 128 & 1.94 & 0.51 & 0.71 & 36.78 & 0.06 & 0.19 & -1.14 & $0.38^{*}$ & $0.56^{*}$ \\
\hline RL13 & 127 & 2.08 & 0.52 & 0.72 & 34.59 & 0.06 & -0.12 & -1.17 & 0.29 & $0.46^{*}$ \\
\hline RL17 & 135 & 2.11 & 0.46 & 0.68 & 32.15 & 0.06 & -0.17 & -1.12 & $0.29^{*}$ & $0.60^{*}$ \\
\hline
\end{tabular}

*significant at $P=0.01$

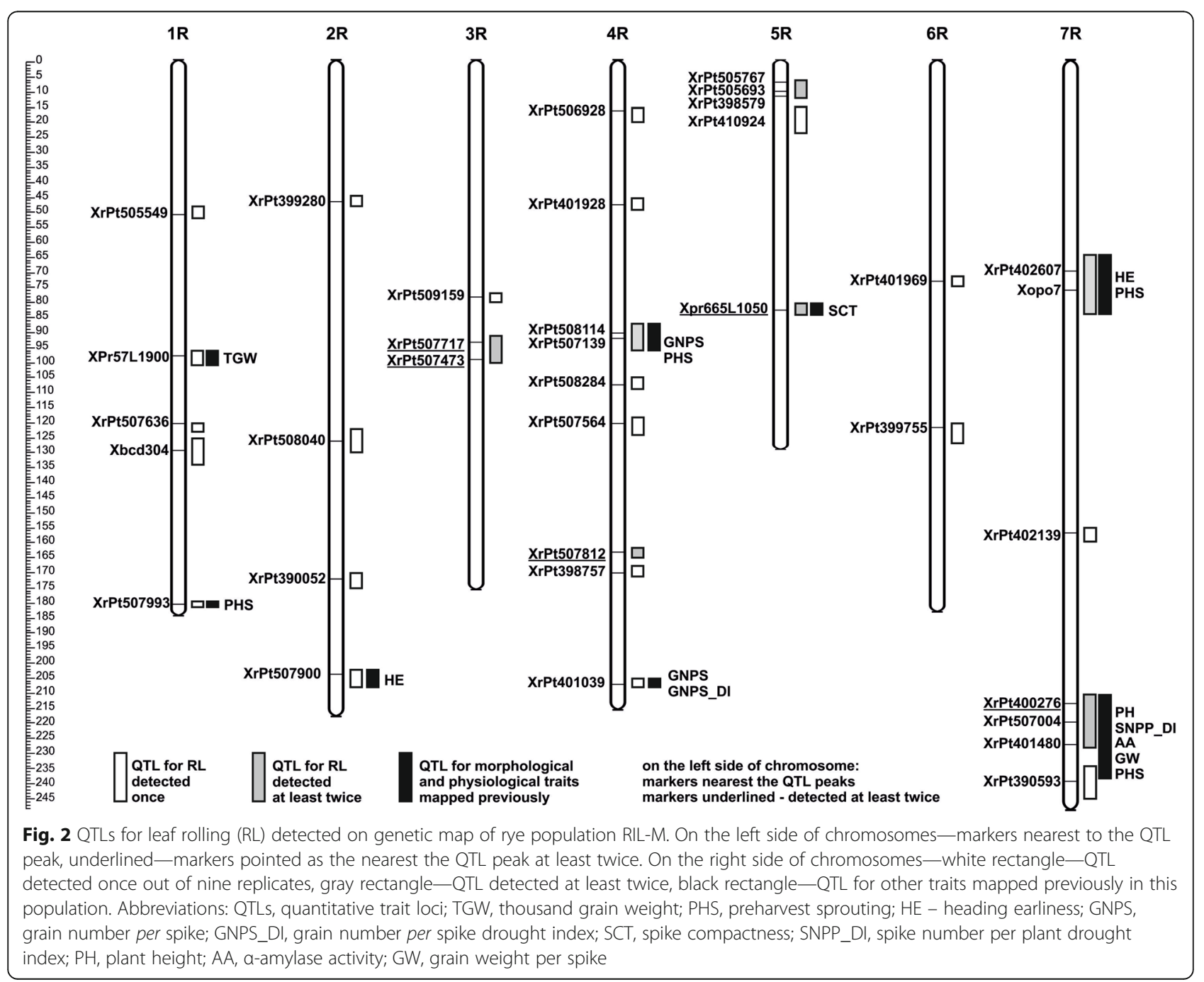


Table 2 QTLs for leaf rolling (RL) in rye mapping population RIL-M, detected at least twice, out of nine replications

\begin{tabular}{|c|c|c|c|c|c|c|c|}
\hline \multirow{2}{*}{$\begin{array}{l}\text { Experiment } \\
\text { replication }\end{array}$} & \multicolumn{3}{|l|}{ QTL position } & \multirow[t]{2}{*}{ LOD } & \multirow[t]{2}{*}{ a } & \multirow[t]{2}{*}{$r^{2}$} & \multirow{2}{*}{$\begin{array}{l}\text { Marker nearest } \\
\text { to the QTL peak }\end{array}$} \\
\hline & Chromosome & Peak [cM] & Interval [cM] & & & & \\
\hline$\overline{R L 10-1}$ & $3 R$ & 94 & $91-96$ & 2.09 & -0.3 & 6.73 & XrPt507717 \\
\hline RL17 & $3 R$ & 95 & $92-98$ & 3.55 & -0.24 & 11.4 & XrPt507717 \\
\hline RL12 & $3 R$ & 98 & 94-101 & 2.25 & -0.22 & 8.17 & XrPt507473 \\
\hline RL08-1 & $3 R$ & 99 & $96-100$ & 4.48 & -0.35 & 12.12 & XrPt507473 \\
\hline RL08-2 & $3 R$ & 99 & 95-101 & 3.12 & -0.31 & 13 & XrPt507473 \\
\hline RL09-1 & $4 \mathrm{R}$ & 90 & $87-96$ & 3.21 & -0.51 & 11.73 & XrPt508114 \\
\hline RL17 & $4 \mathrm{R}$ & 91 & $91-92$ & 3.61 & -0.33 & 11.93 & XrPt507139 \\
\hline RL08-2 & $4 \mathrm{R}$ & 164 & $162-164$ & 3.58 & -0.51 & 15.39 & XrPt507812 \\
\hline RL09-2 & $4 \mathrm{R}$ & 164 & $162-165$ & 2.59 & -0.38 & 7.75 & XrPt507812 \\
\hline RL17 & $5 R$ & 6 & $5-9$ & 4.51 & -0.24 & 11.91 & XrPt505767 \\
\hline RL08-1 & $5 R$ & 9 & $8-12$ & 7.34 & -0.4 & 21 & XrPt505693 \\
\hline RL10-1 & $5 R$ & 12 & $7-12$ & 2.73 & -0.23 & 8.09 & XrPt398579 \\
\hline RL09-1 & $5 R$ & 83 & $81-84$ & 5.38 & -0.35 & 18.59 & Xpr665L1050 \\
\hline RL10-1 & $5 R$ & 83 & $80-84$ & 2.94 & -0.29 & 10.06 & Xpr665L1050 \\
\hline RL08-1 & $5 R$ & 84 & $82-84$ & 4.01 & -0.31 & 9.85 & Xpr665L1050 \\
\hline RL12 & $7 R$ & 69 & $64-76$ & 2.58 & -0.21 & 7.95 & XrPt402607 \\
\hline RL17 & $7 R$ & 76 & $74-84$ & 3.46 & -0.32 & 9.17 & Хopo7 \\
\hline RL09-2 & $7 \mathrm{R}$ & 213 & $212-215$ & 3.67 & -0.26 & 9.41 & XrPt400276 \\
\hline RL17 & $7 \mathrm{R}$ & 215 & $210-216$ & 3.39 & -0.25 & 10.18 & XrPt400276 \\
\hline RL12 & $7 R$ & 221 & $210-221$ & 4.24 & -0.27 & 13.52 & XrPt507004 \\
\hline RL08-2 & $7 R$ & 225 & $215-226$ & 3.64 & -0.35 & 17.84 & XrPt505931 \\
\hline
\end{tabular}

a - additive effect of the maternal allele, $r^{2}$ - variance explained by a QTL

be significant in 7 replications, 19 markers were pointed 6 times, 40 markers were pointed 5 times, 7 markers were pointed 4 times, 8 markers were pointed 3 times, and 10 markers were pointed twice. All intervals containing markers detected in $F$ test included QTL designated by CIM method.

\section{Markers of RL putative homologs}

The set of DArT sequences [19] was screened to find sequences of markers linked to RL. A total of 67 found sequences were directed to BLAST sequence analysis in NCBI nucleotide collection database (https:/www.ncbi.nlm.nih. gov/), using megaBLAST algorithm. Hits exceeding a score of 200 or identity of $95 \%$ and those with known (predicted) identity were chosen. Finally, 12 records met the set criteria (Table 4); one of them was matched to Secale cereale cds, one to Triticum aestivum genomic sequence, and the rest to Aegilops tauschii mRNA.

\section{Co-localization of QTLs}

Some QTL intervals for RL overlapped partially or completely with QTLs for other agronomic traits analyzed previously in this population. There were nine QTLs overlapping most precisely, which means that they had the same markers nearest to the QTL peak and nearly the same intervals (Fig. 2, Table 5).

QTLs for RL were associated with QTLs for morphological traits such as grain number and weight, spike number per plant, compactness of spike, and plant height. Two QTLs of this set were loci controlling drought index (GNPS_DI and SNPP_DI), that is, those that caused differences in trait expression in normal and drought conditions. QTL for RL were also co-localized with two QTLs for heading earliness (2R, 7R), one with $\alpha$-amylase activity QTL (7R) and three for pre-harvest sprouting QTL (1R, 4R, and 7R).

Distal part of chromosome 7RL is a special region with numerous overlapping QTLs: five controlling RL and five engaged in the expression of other traits (Fig. 2, Table 5).

\section{Discussion}

$\mathrm{RL}$ is a phenomenon commonly found in grasses and quite commonly described for field crops, but it has not been studied in rye. As leaf morphology is an important agronomic trait in the breeding of rice [20], the mechanism of RL was of interest, especially to rice researchers. There is no information about the genetic base of 
Table 3 Markers linked to leaf rolling (RL), significant in Fisher's test at $P=0.01$, at least in three replicates

\begin{tabular}{|c|c|c|c|c|c|c|c|c|c|c|c|c|c|c|c|c|c|}
\hline Marker & 1 & 2 & 3 & 4 & 5 & Marker & 1 & 2 & 3 & 4 & 5 & Marker & 1 & 2 & 3 & 4 & 5 \\
\hline XrPt410763 & $7 R$ & 221 & 93 & 22 & 9 & XrPt402377 & $5 R$ & 5 & 96 & 25 & 7 & XrPt390585 & $5 R$ & 121 & 43 & 11 & - \\
\hline XrPt410830 & $7 R$ & 222 & 96 & 22 & 9 & XrPt402405 & $7 R$ & 240 & 66 & 18 & 7 & XrPt398800 & $5 R$ & 121 & 70 & 20 & 5 \\
\hline XrPt346184 & $7 R$ & 224 & 97 & 20 & 8 & XrPt410818 & $3 R$ & 92 & 75 & 19 & 7 & $\underline{\text { XrPt400297 }}$ & $5 R$ & 98 & 50 & 13 & 5 \\
\hline XrPt389258 & $7 R$ & 222 & 96 & 24 & 8 & $\mathrm{XrPt505767}$ & $5 R$ & 6 & 103 & 27 & 7 & XrPt400848 & $3 R$ & 84 & 60 & 15 & \\
\hline XrPt390593 & $7 R$ & 240 & 116 & 24 & 8 & XrPt506147 & $3 R$ & 89 & 72 & 17 & 7 & XrPt401228 & $3 R$ & 84 & 52 & 14 & 5 \\
\hline XrPt390750 & $7 R$ & 251 & 110 & 21 & 8 & XrPt506260 & $7 R$ & 240 & 69 & 17 & 7 & $\underline{\text { XrPt401454 }}$ & $5 R$ & 17 & 69 & 17 & \\
\hline XrPt398579 & $5 R$ & 12 & 103 & 29 & 8 & XrPt507180 & $5 R$ & 12 & 112 & 34 & 7 & XrPt401481 & $3 R$ & 108 & 43 & 13 & \\
\hline XrPt399775 & $7 R$ & 233 & 68 & 14 & 8 & XrPt507500 & $5 R$ & 5 & 103 & 27 & 7 & XrPt402245 & $5 R$ & 16 & 76 & 20 & J \\
\hline XrPt400252 & $7 R$ & 224 & 90 & 17 & 8 & $\underline{\mathrm{XrPt507717}}$ & $3 R$ & 94 & 82 & 18 & 7 & $\underline{\text { XrPt402531 }}$ & $5 R$ & 125 & 47 & 16 & \\
\hline XrPt400319 & $3 R$ & 89 & 63 & 13 & 8 & XrPt507948 & $5 R$ & 0 & 83 & 23 & 7 & XrPt410924 & $5 R$ & 15 & 92 & 22 & 5 \\
\hline XrPt400793 & $7 R$ & 233 & 68 & 14 & 8 & XrPt508061 & $7 R$ & 72 & 68 & 14 & 7 & XrPt411109 & $5 R$ & 113 & 57 & 16 & J \\
\hline XrPt401200 & $7 R$ & 227 & 114 & 19 & 8 & Xpr665L1050 & $5 R$ & 84 & 85 & 22 & 6 & XrPt411252 & $5 R$ & 17 & 76 & 20 & \\
\hline XrPt401372 & $7 R$ & 233 & 75 & 15 & 8 & XrPt346908 & $3 R$ & 78 & 56 & 14 & 6 & XrPt411320 & $5 R$ & 119 & 56 & 16 & 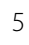 \\
\hline XrPt401480 & $7 R$ & 227 & 94 & 16 & 8 & XrPt347574 & $7 R$ & 65 & 71 & 16 & 6 & XrPt505219 & $7 \mathrm{R}$ & 65 & 47 & 17 & 5 \\
\hline XrPt401828 & $7 R$ & 247 & 121 & 24 & 8 & XrPt347998 & $5 R$ & 14 & 92 & 22 & 6 & XrPt505693 & $5 R$ & 8 & 89 & 29 & 5 \\
\hline XrPt402262 & $7 R$ & 247 & 109 & 21 & 8 & $\underline{\mathrm{XrPt} 398502}$ & $3 R$ & 100 & 69 & 17 & 6 & XrPt505721 & $5 R$ & 113 & 57 & 16 & 5 \\
\hline XrPt410884 & $7 R$ & 233 & 68 & 14 & 8 & XrPt398519 & $7 R$ & 65 & 71 & 16 & 6 & XrPt506001 & $5 \mathrm{R}$ & 17 & 76 & 20 & 5 \\
\hline XrPt505215 & $3 R$ & 91 & 67 & 13 & 8 & $\underline{\text { XrPt401081 }}$ & $3 R$ & 100 & 69 & 17 & 6 & XrPt506821 & $5 R$ & 102 & 48 & 13 & 5 \\
\hline XrPt505523 & $7 R$ & 233 & 68 & 14 & 8 & XrPt401795 & $7 R$ & 65 & 71 & 16 & 6 & $\underline{\mathrm{XrPt} 506905}$ & $3 R$ & 79 & 51 & 14 & 5 \\
\hline XrPt505864 & $7 R$ & 224 & 97 & 20 & 8 & XrPt402589 & $3 R$ & 76 & 57 & 15 & 6 & XrPt507369 & $3 R$ & 84 & 52 & 14 & J \\
\hline XrPt505931 & $7 R$ & 224 & 97 & 20 & 8 & $\underline{\mathrm{XrPt} 402607}$ & $7 R$ & 68 & 74 & 17 & 6 & XrPt507926 & $5 R$ & 98 & 50 & 13 & 5 \\
\hline XrPt506494 & $7 R$ & 222 & 96 & 20 & 8 & $\underline{\text { XrPt410783 }}$ & $5 R$ & 12 & 92 & 22 & 6 & XrPt507953 & $5 R$ & 122 & 72 & 20 & 5 \\
\hline XrPt506607 & $3 R$ & 91 & 67 & 13 & 8 & XrPt411522 & $5 R$ & 121 & 57 & 14 & 6 & XrPt508905 & $5 R$ & 98 & 50 & 13 & 5 \\
\hline XrPt506764 & $7 R$ & 224 & 95 & 19 & 8 & XrPt505593 & $3 R$ & 89 & 77 & 19 & 6 & XrPt508925 & $5 R$ & 7 & 82 & 24 & 5 \\
\hline XrPt507754 & $7 R$ & 222 & 96 & 21 & 8 & XrPt506874 & $3 R$ & 103 & 48 & 14 & 6 & XrPt509159 & $3 R$ & 79 & 62 & 17 & 5 \\
\hline XrPt507936 & $7 R$ & 222 & 91 & 21 & 8 & $\mathrm{XrPt507004}$ & $7 R$ & 220 & 81 & 18 & 6 & Xscm141 & $5 R$ & 79 & 53 & 12 & 5 \\
\hline XrPt508559 & $7 R$ & 72 & 86 & 16 & 8 & XrPt507462 & $3 R$ & 76 & 57 & 15 & 6 & Xscsz877L950 & $5 R$ & 121 & 59 & 16 & 5 \\
\hline XrPt508837 & $7 R$ & 222 & 96 & 20 & 8 & $\underline{\mathrm{XrPt507473}}$ & $3 R$ & 99 & 95 & 25 & 6 & XrPt346583 & $5 R$ & 108 & 36 & 11 & 4 \\
\hline Xоро7 & $7 R$ & 76 & 82 & 18 & 7 & $\underline{\text { XrPt508197 }}$ & $5 R$ & 5 & 98 & 27 & 6 & XrPt39 & $7 R$ & 208 & 48 & 14 & 4 \\
\hline Xpr57L470 & $5 R$ & 101 & 68 & 15 & 7 & XrPt509647 & $3 R$ & 78 & 56 & 14 & 6 & XrPt399654 & $7 R$ & 209 & 44 & 12 & 4 \\
\hline XrPt117252 & $3 R$ & 89 & 72 & 17 & 7 & Amy3.2 & $5 R$ & 128 & 50 & 13 & 5 & XrPt411020 & $5 R$ & 121 & 55 & 15 & 4 \\
\hline XrPt119718 & $5 R$ & 84 & 51 & 11 & 7 & Xpr665ட430 & $3 R$ & 81 & 41 & 10 & 5 & XrPt411184 & $5 R$ & 18 & 59 & 16 & 4 \\
\hline XrPt120990 & $3 R$ & 89 & 72 & 17 & 7 & XrPt346755 & $5 R$ & 17 & 76 & 20 & 5 & XrPt505437 & $7 R$ & 208 & 50 & 13 & 4 \\
\hline XrPt345439 & $3 R$ & 89 & 72 & 17 & 7 & 5892 & $5 R$ & 17 & 76 & 20 & 5 & 051 & $5 R$ & 106 & 43 & 12 & 4 \\
\hline XrPt348093 & $3 R$ & 89 & 72 & 17 & 7 & XrPt346946 & $5 R$ & 17 & 76 & 20 & 5 & XrPt346779 & $6 \mathrm{R}$ & 59 & 35 & 16 & 3 \\
\hline XrPt389261 & $5 R$ & 12 & 108 & 32 & 7 & XrPt346980 & $5 R$ & 17 & 76 & 20 & 5 & XrPt347114 & $6 \mathrm{R}$ & 63 & 33 & 16 & 3 \\
\hline XrPt389585 & $7 R$ & 216 & 81 & 17 & 7 & XrPt347072 & $5 R$ & 17 & 76 & 20 & 5 & XrPt389711 & $7 \mathrm{R}$ & 207 & 38 & 13 & 3 \\
\hline XrPt389895 & $5 R$ & 5 & 103 & 27 & 7 & XrPt347212 & $5 R$ & 16 & 79 & 21 & 5 & XrPt390442 & $6 \mathrm{R}$ & 59 & 35 & 16 & 3 \\
\hline XrPt389959 & $7 R$ & 210 & 96 & 23 & 7 & XrPt347454 & $5 R$ & 17 & 76 & 20 & 5 & XrPt400509 & $7 R$ & 207 & 34 & 11 & 3 \\
\hline XrPt390362 & $5 R$ & 6 & 96 & 25 & 7 & XrPt347809 & $5 R$ & 17 & 76 & 20 & 5 & XrPt400732 & $7 R$ & 202 & 38 & 13 & 3 \\
\hline XrPt398627 & $3 R$ & 89 & 72 & 17 & 7 & XrPt389454 & $3 R$ & 81 & 53 & 14 & 5 & XrPt401523 & $7 R$ & 207 & 48 & 12 & 3 \\
\hline XrPt400276 & $7 R$ & 214 & 96 & 23 & 7 & XrPt389759 & $5 R$ & 107 & 38 & 12 & 5 & XrPt509176 & $7 R$ & 207 & 48 & 12 & 3 \\
\hline XrPt401754 & $7 R$ & 73 & 55 & 12 & 7 & XrPt389815 & $3 R$ & 81 & 53 & 14 & 5 & & & & & & \\
\hline
\end{tabular}


Table 4 DArTs linked to leaf rolling (RL) with known sequences, annotated in NCBI

\begin{tabular}{|c|c|c|c|c|c|c|c|c|c|c|}
\hline \multicolumn{3}{|l|}{ Marker } & \multicolumn{8}{|l|}{ NCBI annotation } \\
\hline Marker & $\begin{array}{l}\text { DArT } \\
\text { sequence } \\
\text { length (bp) }\end{array}$ & Chrom. & Description & Species & $\begin{array}{l}\text { Max } \\
\text { score }\end{array}$ & $\begin{array}{l}\text { Total } \\
\text { score }\end{array}$ & $\begin{array}{l}\text { Query } \\
\text { cover }\end{array}$ & E value & Identity & Accession \\
\hline $\mathrm{XrPt506905}$ & 665 & $3 R$ & $\begin{array}{l}\text { subtilisin-like protease } \\
\text { SBT2.2 (LOC109761160), } \\
\text { mRNA }\end{array}$ & $\begin{array}{l}\text { Aegilops tauschii } \\
\text { subsp. tauschii }\end{array}$ & 97 & 97 & $8 \%$ & $6.00 \mathrm{E}-16$ & $95 \%$ & XM_020319955.1 \\
\hline XrPt507717 & 678 & $3 R$ & $\begin{array}{l}\text { protein LIKE COV } 2 \\
\text { (LOC109734806), mRNA }\end{array}$ & $\begin{array}{l}\text { Aegilops tauschii } \\
\text { subsp. tauschii }\end{array}$ & 279 & 279 & $24 \%$ & $5.00 \mathrm{E}-71$ & $96 \%$ & XM_020293989.1 \\
\hline XrPt507473 & 599 & $3 R$ & $\begin{array}{l}\text { Fhb1 region genomic } \\
\text { sequence }\end{array}$ & $\begin{array}{l}\text { Triticum aestivum } \\
\text { cv Sumai } 3\end{array}$ & 60 & 60 & $5 \%$ & 7.00E-05 & $97 \%$ & KX907434.1 \\
\hline $\begin{array}{l}\text { XrPt401081 } \\
\text { XrPt398502 }\end{array}$ & 545 & $3 R$ & $\begin{array}{l}\text { jasmonate O-methyltransferase- } \\
\text { like (LOC109744228), mRNA }\end{array}$ & $\begin{array}{l}\text { Aegilops tauschii } \\
\text { subsp. tauschii }\end{array}$ & 359 & 692 & $86 \%$ & $5.00 \mathrm{E}-95$ & $95 \%$ & XM_020303320.1 \\
\hline XrPt508197 & 720 & $5 R$ & RGA1-G gene, complete cds & Secale cereale & 220 & 358 & $19 \%$ & 4.00E-53 & $96 \%$ & KT725818.1 \\
\hline XrPt410783 & 463 & $5 R$ & $\begin{array}{l}\text { cancer-related nucleoside- } \\
\text { triphosphatase (LOC109750338), } \\
\text { mRNA }\end{array}$ & $\begin{array}{l}\text { Aegilops tauschii } \\
\text { subsp. tauschii }\end{array}$ & 527 & 624 & $75 \%$ & $1.00 \mathrm{E}-145$ & $94 \%$ & XM_020309304.1 \\
\hline XrPt401454 & 218 & $5 R$ & $\begin{array}{l}\text { LRR receptor-like serine/ } \\
\text { threonine-protein kinase } \\
\text { FEI } 1 \text { (LOC109774869), } \\
\text { mRNA }\end{array}$ & $\begin{array}{l}\text { Aegilops tauschii } \\
\text { subsp. tauschii }\end{array}$ & 57 & 57 & $13 \%$ & $3.00 \mathrm{E}-04$ & $100 \%$ & XM_020333663.1 \\
\hline XrPt400297 & 773 & $5 R$ & $\begin{array}{l}\text { serine/threonine-protein } \\
\text { kinase AFC1-like } \\
\text { (LOC109779169), } \\
\text { transcript variant X4, } \\
\text { mRNA }\end{array}$ & $\begin{array}{l}\text { Aegilops tauschii } \\
\text { subsp. tauschii }\end{array}$ & 228 & 282 & $19 \%$ & 2.00E-55 & $100 \%$ & XM_020337784.1 \\
\hline XrPt402531 & 422 & $5 R$ & $\begin{array}{l}\text { transcription factor } \\
\text { bHLH79-like } \\
\text { (LOC109737374), mRNA }\end{array}$ & $\begin{array}{l}\text { Aegilops tauschii } \\
\text { subsp. tauschii }\end{array}$ & 99 & 99 & $13 \%$ & $1.00 \mathrm{E}-16$ & $98 \%$ & XM_020296517.1 \\
\hline XrPt402607 & 551 & $7 R$ & $\begin{array}{l}\text { polyadenylate-binding } \\
\text { protein-interacting } \\
\text { protein 7-like } \\
\text { (LOC109738116), } \\
\text { transcript variant } \\
\text { X4, mRNA }\end{array}$ & $\begin{array}{l}\text { Aegilops tauschii } \\
\text { subsp. tauschii }\end{array}$ & 470 & 470 & $60 \%$ & $2.00 \mathrm{E}-128$ & $93 \%$ & XM_020297211.1 \\
\hline XrPt401480 & 561 & $7 \mathrm{R}$ & $\begin{array}{l}\text { tubby-like F-box protein } \\
12 \text { (LOC109763585), } \\
\text { transcript variant X3, } \\
\text { mRNA }\end{array}$ & $\begin{array}{l}\text { Aegilops tauschii } \\
\text { subsp. tauschii }\end{array}$ & 601 & 601 & $65 \%$ & $8.00 \mathrm{E}-168$ & $96 \%$ & XM_020322436.1 \\
\hline XrPt390593 & 451 & $7 R$ & $\begin{array}{l}\text { vegetative cell wall } \\
\text { protein gp1-like } \\
\text { (LOC109748681), } \\
\text { transcript variant } \\
\text { X4, mRNA }\end{array}$ & $\begin{array}{l}\text { Aegilops tauschii } \\
\text { subsp. tauschii }\end{array}$ & 57 & 57 & $6 \%$ & $6.00 \mathrm{E}-04$ & $100 \%$ & XM_020307705.1 \\
\hline
\end{tabular}

Bolded-markers nearest to the QTL peak

molecular mechanisms involved in leaf shape in rye, except one report regarding QTLs controlling leaf area [21].

A study similar to ours has been conducted on tetraploid wheat [4]; however, we did not study RL as the reaction to water deficit. The research on drought resistance in durum wheat $\times$ wild emmer wheat recombinant inbred line population allowed to detect 11 significant QTLs associated with flag RL, mapped on chromosomes: 1A, 2A, 2B, 4B, 5A, 5B, 6A, 6B, 7A, and $7 \mathrm{~B}$ [4]. Three of these QTLs were found to be environment responsive.
We detected 43 QTLs, grouped into 28 intervals, which confirms the multigenic base of the trait stated for wheat and rice. Studies on rice showed that no fewer than 70 genes/QTLs for RL have been mapped or cloned till now [11]. Four QTLs for RL were stable in different environments. Due to many QTLs for RL were detected once, their genotypexenvironment interaction (GEI) could be inferred. GEI is a common characteristic for quantitative traits. For possible breeding purposes (like marker Assisted Selection - MAS), QTLs that are more environment-specific should be treated with caution and 
Table 5 QTLs for leaf rolling (RL) in rye mapping population RIL-M, coinciding with QTLs for other traits, mapped previously in this population

\begin{tabular}{|c|c|c|c|c|c|c|c|}
\hline Chrom. & Coinciding QTLs & $\begin{array}{l}\text { Marker nearest to } \\
\text { the QTL peak }\end{array}$ & QTL peak [cM] & QTL interval [cM] & LOD & a & $r^{2}$ \\
\hline \multirow[t]{2}{*}{$1 \mathrm{R}$} & RL08-1 & Xpr57L1900 & 98 & $96-101$ & 2.7 & 0.33 & 8.61 \\
\hline & TGW09 & & 98 & 98-102 & 3.03 & -1.94 & 9.42 \\
\hline \multirow[t]{2}{*}{$1 \mathrm{R}$} & RL12 & XrPt507993 & 181 & 180-182 & 2.8 & 0.24 & 8.57 \\
\hline & PHS08 & & 181 & $178-183$ & 3.02 & -6.05 & 8.37 \\
\hline \multirow[t]{2}{*}{$2 R$} & HE08 & Xrpt507900 & 202 & $200-206$ & 3.33 & 0.55 & 8.74 \\
\hline & RL08-1 & & 204 & $202-208$ & 3.1 & -0.31 & 9.63 \\
\hline \multirow[t]{3}{*}{$4 \mathrm{R}$} & GNPS08 & XrPt508114 & 89 & $87-91$ & 3.05 & 6.77 & 10.8 \\
\hline & PHS10 & & 89 & $85-106$ & 2.69 & -8.64 & 9.2 \\
\hline & RL09-1 & & 90 & $87-96$ & 3.21 & -0.51 & 11.73 \\
\hline \multirow[t]{3}{*}{$4 \mathrm{R}$} & GNPS08 & XrPt401039 & 207 & $206-208$ & 4.47 & -3.94 & 12.54 \\
\hline & RL10-2 & & 207 & $205-208$ & 3.36 & -0.36 & 10.77 \\
\hline & GNPS_DI & & 207 & $205-210$ & 2.73 & 12.76 & 15.45 \\
\hline \multirow[t]{4}{*}{$5 R$} & SCT09 & Xpr665L1050 & 81 & $73-84$ & 2.8 & -0.1 & 12.02 \\
\hline & RL09-1 & & 81 & $81-84$ & 5.38 & -0.35 & 18.59 \\
\hline & RL10-1 & & 83 & $80-84$ & 2.94 & -0.29 & 10.06 \\
\hline & RL-08-1 & & 84 & $82-84$ & 4.01 & -0.31 & 9.85 \\
\hline \multirow[t]{3}{*}{$7 \mathrm{R}$} & RL17 & Xopo7 & 76 & $74-84$ & 3.46 & -0.32 & 9.17 \\
\hline & PHSO7 & & 77 & $69-87$ & 2.74 & 13.76 & 18.49 \\
\hline & HE09 & & 84 & 74-95 & 2.3 & 0.83 & 16.97 \\
\hline \multirow[t]{3}{*}{$7 \mathrm{R}$} & SNPP_DI & XrPt507004 & 219 & $214-228$ & 1.85 & -8.38 & 9.9 \\
\hline & PH08 & & 220 & $216-222$ & 2.76 & 4.15 & 8.31 \\
\hline & RL12 & & 221 & $210-221$ & 4.24 & -0.27 & 13.52 \\
\hline \multirow[t]{4}{*}{$7 \mathrm{R}$} & AA09 & XrPt401480 & 227 & $226-238$ & 8.25 & 1.21 & 22.85 \\
\hline & RL10-1 & & 227 & $227-228$ & 3.68 & -0.28 & 11.51 \\
\hline & PHS08N & & 228 & $227-238$ & 5.17 & 9.89 & 16.64 \\
\hline & GW09 & & 229 & $224-229$ & 2.11 & -0.09 & 6.62 \\
\hline
\end{tabular}

a - additive effect of the maternal allele, $r^{2}$ - variance explained by a QTL

TGW thousand grain weight, PHS preharvest sprouting, HE heading earliness, GNPS grain number per spike, GNPS_DI grain number per spike drought index, SCT spike compactness, SNPP_DI spike number per plant drought index, $P H$ plant hight, $A A$ a-amylase activity, $G W$ grain weight per spike

more attention should be paid to the repetitive QTLs. However, QTLs of varying manifestation, dependent on the environmental influence are also interesting for cognitive purposes.

In this study, we focused on the analysis of RL per se, without linking this trait with the response to drought. However, co-localization of two QTLs for RL with QTLs for drought index of grain number per spike (4R) and spike number per plant (7R) revealed in other experiments [18] indicates the relationship of detected loci with adaptive mechanisms to drought-related stress conditions.

Furthermore, 5 out of 11 QTLs for RL mapped on tetraploid wheat were co-localized with QTLs associated with plant productivity [4]. RL was also found to be associated with plant height in four regions $(2 \mathrm{~B}, 4 \mathrm{~B}, 6 \mathrm{~A}$, and 7A) and with heading earliness (days from planting to heading) in two intervals on chromosomes $4 \mathrm{~B}$ and $5 \mathrm{~A}$ [4]. We also found nine QTLs co-localized with QTLs for other agronomic traits mapped in the same population [17], such as grain number and weight, spike number per plant, compactness of spike and plant height. QTLs for RL were also found to be co-localized with two QTLs for heading earliness ( $2 R$ and $7 R$ ), one with $\alpha$-amylase activity QTL (7R), and three with preharvest sprouting QTLs (1R, 4R, and 7R) [16].

Additional confirmation of the association between loci engaged in controlling RL and QTLs responsible for different agronomic traits is the result of comparing markers linked with RL and markers for nine features studied in the other, unrelated rye RILs' mapping population $541 \times$ Ot1-3 [21]. There were loci for plant height, 
stem thickness, spike length, awn length, heading date, thousand grain weight, grain length, leaf area, and chlorophyll content localized on the DArT-based high-density map of this population.

Each of the nine traits was characterized by some markers common with these, linked to RL in our population. There were 45 such markers distributed throughout the five chromosomes (3R, 4R, 5R, 6R, and 7R). Among them three DArTs from 7R were common for RL and leaf size (XrPt505931, XrPt389959, and XrPt400276). Seven DArTs were linked to RL and chlorophyll content: three from 5R (XrPt389759, XrPt346583, and XrPt505721) and four from 7R chromosome (XrPt402607, XrPt398519, $\mathrm{XrPt} 347574$, and $\mathrm{XrPt} 401795)$. All these relationships suggest very strong linkages and/or pleiotropic effects of many genes, which remains in agreement with previous results for rye [16, 17, 21], wheat [4], and rice [22].

All DArTs significantly linked to RL were subjected to screening the DArT sequences database, followed by NCBI database blasting, in order to find the homologs. A total of 12 records with a known identity were found; majority of rye DArTs sequences were most similar to Aegilops tauschii mRNAs. Only one of them was matched to Secale cereale cds of resistant gene analog (putative disease resistance gene), and one to Triticum aestivum genomic sequence-also connected with resistance, in this case to fusarium head blight (FHB).

Sequence of DArT XrPt506905 from 3R showed similarity to predicted gene, namely, subtilisin-like protease. Subtilisin-like proteases (subtilases) are serine proteases and constitute the largest group of peptidases. Although several subtilases have been identified in plants (e.g., about 60 subtilase genes are known in Oryza sativa and Arabidopsis thaliana), most of their functions in plants remain unknown [23] (and bibliography therein). It is likely that subtilases contribute significantly to the developmental processes and signaling cascades in plants (Rautengarten et al. 2005, after [23]). For instance, the loss-of-function mutation in ALE1 leads to abnormal leaf shape [24]. Marker XrPt506905, which is a predicted gene for subtilisin-like protein, in addition to the linkage with RL also showed a relationship with awn and grain length [21].

DArT XrPt507717, nearest to the peak of a QTL for RL from 3R, revealed similarity to protein-like COV2 mRNA. The role of COV2, inferred from the sequence or structural similarity to COV1 is stem vascular tissue pattern formation. COV1 is predicted to be an integral membrane protein that may be involved in the perception or transport of a signaling molecule that negatively regulates the differentiation of vascular tissue in the developing stem of Arabidopsis [25]. Marker XrPt507717, in addition to the linkage with RL also showed a relationship with awn length [21].
Two DArTs, XrPt401081 and XrPt398502 from 3R, has sequences homologous to jasmonate $O$-methyltransferase. This enzyme catalyzes the methylation of jasmonate into methyljasmonate, a plant volatile that acts as an important cellular regulator mediating diverse developmental processes and defense responses (http:// www.uniprot.org/uniprot/Q9AR07). It is involved in the pathway of oxylipin biosynthesis, which is a part of lipid metabolism. To this end, 28 differentially expressed proteins related to rolled leaf traits were isolated and identified. Some of the proteins and genes detected are involved in lipid metabolism, which is related to the development of bulliform cells, such as phosphoinositide phospholipase C, Mgll, and At4g26790 [12].

Sequence of next DArT from 3R, XrPt507473, proved to be similar to Fhb1, a major FHB-resistant gene. Fhb1 was fine mapped on the distal segment of chromosome 3BS of spring wheat (Triticum aestivum L.). One of the recent studies has reported that wheat $F h b 1$ encodes a chimeric lectin with agglutinin domains and a pore-forming toxin-like domain conferring resistance to FHB [26]. Marker XrPt507473, in addition to the linkage with RL, also showed a relationship with awn length and stem thickness [21].

XrPt401454 from 5R showed homology to LRR receptor-like serine/threonine-protein kinase FEI11 mRNA. FEI1 is involved in the signaling pathway that regulates cell wall function, including cellulose biosynthesis, likely via an 1-aminocyclopropane-1-carboxylic acid (ACC)-mediated signal; a precursor of ethylene (http://www.uniprot.org/uniprot/COLGF4). To date, 13 genes associated with rice RL have been isolated or cloned. The cytological mechanism of RL has been found to be largely related to the abnormal development of bulliform cells. NRL1 encodes cellulose synthase and plays a positive role in the regulation of bulliform cell development. In mutant rice plants that lack this gene, shrinkage is found in the area of the bulliform cells, thereby causing inward rolling of rice leaves [12].

Sequence of XrPt402531 from 5R demonstrated high similarity to predicted $A$. tauschii transcription factor bHLH79 (basic helix-loop-helix protein 79). The function of this factor is unknown; however, several transcription factors are known to be engaged in the establishment of abaxial/adaxial leaf polarity. For example, mutation in SLL1/RL9, a member of the KANADI family, encoding a transcription factor [27], leads to the failure of programmed cell death of abaxial mesophyll cells and the suppression of the differentiation of the abaxial cells, and finally to generate adaxially rolled leaves. ROC5 encodes a protein containing a leucine zipper domain, homologous to GLABRA2 in Arabidopsis which results in the negative regulation in the development of the bulliform cells. The number and size of the bulliform cells increased when ROC5 was knocked out, thereby leading to the generation 
of adaxially rolled leaves, whereas co-suppression of ROC5 resulted in abaxial RL [28].

Overexpression of a rice gene OsLBD3-7 that encodes a LBD family transcription factor promoted narrow and adaxially rolled leaves by decreasing the size and number of bulliform cells. OsLBD3-7 also upregulated the expression of negative regulators of bulliform cells, which implies that OsLBD3-7 acts as a suppressor of bulliform cell development [23].

The other rice gene ACL1 encodes an unknown protein with a conserved functional domain; OsZHD1 encodes a domain transcription factor with homologous zinc finger structure. These genes also play a positive role in the regulation of bulliform cell development, and overexpression of these two genes results in an increased number of bulliform cells, thereby causing outward rolling of rice leaves [12, 29].

XrPt402607 from 7R, marker nearest to the peak of QTL for RL and also marker linked with grain length and chlorophyll content [21], showed homology to predicted polyadenylate-binding protein-interacting protein 7-like gene. The poly(A) binding proteins (PABP) play an important role in the regulation of translation; however, the role of this particular factor is unknown.

Next marker from 7R, the peak of QTL for RL, DArT $\mathrm{XrPt} 401480$, seems to be homologous to the predicted gene encoding tubby-like F-box protein 12 . Plants include a large number of tubby-like proteins (TLPs/ TULPs). For example, there are 11 members of the tubby gene family in Arabidopsis [30], 14 in rice [31], 11 in poplar [32], 4 in wheat [33], and 8 in sorghum (http:// www.ncbi.nlm.nih.gov). The existence of multiple TLPs implies their vital function in plants. F-box proteins regulate diverse cellular processes, including cell cycle transition, transcriptional regulation, and signal transduction. Lai et al. [30] have demonstrated that AtTLP9 interacts with ASK1 (Arabidopsis Skp1-like 1). According to them, F-box domain containing plant TLPs acting as transcription regulators should have cellular function activities of F-box proteins in signal transduction. AtTLP9 might participate in the abscisic acid signaling pathway [30]. The other example of F-box protein regulating plant growth and development include TIR1 acting in response to auxin [34].

The function of tubby-like F-box protein 12 encoded by genes of Aegilops tauschii and Brachypodium distach-

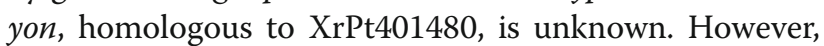
some rice TLPs, especially OsTLP12, were probably involved in the abscisic acid and gibberellin signaling processes. This role might also be attributed to rye TLP12, because the same DArT was pointed as a marker linked to plant height [21].

XrPt390593 sequence from 7R was similar to vegetative cell wall protein gp1-like mRNA. The nature of cell wall proteins is as varied as the many functions of plant cell walls. Majority of the cell wall proteins are cross-linked into the cell wall and probably have structural functions. If this protein was associated with bulliform and/or hypodermis cells it might have an effect on RL, because these two types of cells are involved in RL in higher plants [1].

Although the roles described for the aforementioned markers as the potential genes that control RL are likely, their functions and association with RL and other traits should be verified in expression tests and will be studied during further research.

\section{Conclusion}

The first localization of loci controlling leaf rolling (RL) on high density consensus genetic map of rye confirms the multigenic base of the trait stated for wheat and rice. Four stable quantitative QTLs on chromosomes 3R, 5R, and 7R were identified. Coinciding QTLs for RL and agronomic traits (e.g. drought tolerance) suggested pleiotropic effects of genes engaged in leaf rolling control. Four reproducible QTLs may be interesting for breeding purposes. The composite QTL analyze, the knowledge of the sequence of markers strongly linked to RL and the comparison of their homology with sequences data of related species allowed to indicate rye candidate genes controlling RL process.

\section{Additional files}

Additional file 1: Matrix of coefficient correlation of leaf rolling $(R L)$ in nine experimental variants, assessed in rye mapping population RIL-M. (XLSX $11 \mathrm{~kb}$ )

Additional file 2: Phenotypic data of rye mapping population RIL-M. (XLSX 66 kb)

\begin{abstract}
Abbreviations
AA: a-amylase activity; bHLH: Basic helix-loop-helix protein; DArT: Diversity array technology; GNPS: Grain number per spike; GNPS_DI: Grain number per spike drought index; GW: Grain weight per spike; HE: Heading earliness; MAS: Marker assisted selection; PH: Plant height; PHS: Pre-harvest sprouting; QTLS: Quantitative trait loci; RIL: Recombinant inbred line; RL: Leaf rolling; SCT: Spike compactness; SNPP_DI: Spike number per plant drought index; TGW: Thousand grain weight; TLP: Tubby-like proteins
\end{abstract}

\section{Acknowledgements \\ Thanks to Elżbieta Pauker, Henryk Pauker and Jarosław Myśków for technical assistance. \\ Funding \\ The study was financially supported by Ministry of Science and Higher Education, Republic of Poland under grant No 518070173171-01/18. The funding body played no role in the design of the study and collection, analysis, and interpretation of data and in writing the manuscript.}

\section{Availability of data and materials}

The raw mapping data are available in the article by Milczarski et al. [15] and the phenotypic data are submitted as the supplementary material in Additional file 2 . 


\section{Authors' contributions}

Conceived and designed the experiments: BM. Performed the experiments: BM, NL. Analyzed the data: BM, MG, NL, IC-M. Wrote the paper: BM. Critically revised the paper: SS. Proportions of authors' contribution: 6:1:1:1:1 (BM, MG, NL, IC-M, SS). All authors read and approved the final version of the manuscript.

\section{Ethics approval and consent to participate}

This study has not directly involved humans and animals. The plant material belonged to the West-Pomeranian University of Technology, Szczecin; the mapping population was bred by the authors. No special permissions and/or licences were necessary.

\section{Consent for publication}

Not applicable

\section{Competing interests}

The authors declare that they have no competing interests.

\section{Publisher's Note}

Springer Nature remains neutral with regard to jurisdictional claims in published maps and institutional affiliations.

\section{Author details}

'Department of Plant Genetics, Breeding and Biotechnology, West-Pomeranian University of Technology, Szczecin, ul. Słowackiego 17, 71-434 Szczecin, Poland. ${ }^{2}$ The Franciszek Górski Institute of Plant Physiology, Polish Academy of Science, ul. Niezapominajek 21, 30-239 Cracow, Poland.

Received: 22 March 2018 Accepted: 31 July 2018

\section{Published online: 09 August 2018}

\section{References}

1. Kadioglu A, Terzi R. A dehydration avoidance mechanism: leaf rolling. Bot Rev. 2007;73(4):290-302.

2. Fang L, Zhao F, Cong Y, Sang X, Du Q, Wang D, et al. Rolling-leaf14 is a 2OG-Fe (II) oxygenase family protein that modulates rice leaf rolling by affecting secondary cell wall formation in leaves. Plant Biotechnol J. 2012; 10(5):524-32.

3. Clarke JM. Effect of leaf rolling on leaf water loss in Triticum Spp. Can J Plant Sci. 1986;66:885-91.

4. Peleg Z, Fahima T, Krugman T, Abbo S, Yakir D, Korol AB, et al. Genomic dissection of drought resistance in durum wheat $\times$ wild emmer wheat recombinant inbreed line population. Plant Cell Environ. 2009;32(7):758-79.

5. Sarieva GE, Kenzhebaeva SS, Lichtenthaler HK. Adaptation potential of photosynthesis in wheat cultivars with a capability of leaf rolling under high temperature conditions. Russ J Plant Physiol. 2010:57(1):28-36. Available from: http://link.springer.com/10.1134/S1021443710010048

6. Araya A, Belay T, Hussein T. Variation between Ethiopian and north American barley varieties (Hordeum vulgare) in response to Russian wheat aphid (Diuraphis noxia) populations. J Insect Sci. 2014;14:40.

7. O'Toole JC, Cruz RT. Response of Leaf Water Potential, Stomatal Resistance, and Leaf Rolling to Water Stress. Plant Physiol. 1980;65(3):428-32. Available from: http://www.plantphysiol.org/cgi/doi/10.1104/pp.65.3.428

8. Wang S, Basten CJ, Zeng BZ. Windows QTL Cartographer 2.5. Raleigh: Department of Statistics, North Carolina State University; 2012. Available from: http://statgen.ncsu.edu/qtlcart/WQTLCart.htm

9. Moon J, Hake S. How a leaf gets its shape. Curr Opin Plant Biol. 2011;14(1): 24-30. Available from: https://doi.org/10.1016/j.pbi.2010.08.012

10. Liu X, Li M, Liu K, Tang D, Sun M, Li Y, et al. Semi-rolled Leaf2 modulates rice leaf rolling by regulating abaxial side cell differentiation. J Exp Bot. 2016;67(8):2139-50.

11. Zhang Q, Zheng T, Hoang L, Wang C, Nafisah, Joseph C, Zhang W, Xu J, Li Z. Joint mapping and allele mining of the rolled leaf trait in rice (Oryza sativa L.) PLoS One. 2016:11(7):1-15.

12. Zhu Q, Yu S, Chen G, Ke L, Pan D. Analysis of the differential gene and protein expression profile of the rolled leaf mutant of transgenic rice (Oryza sativa L.). PLoS One. 2017;12(7):e0181378.

13. Myśków B, Masojć P, Banek-Tabor A, Szołkowski A. Genetic diversity of inbred rye lines evaluated by RAPD analysis. J Appl Genet. 2001;42(1):1-14
14. Korol A, Mester D, Frenkel ZRY. Methods for genetic analysis in the Triticeae. In: Feuillet C, Muehlbauer G, editors. Genetics and genomics of the Triticeae. New York: Springer Science + Business Media; 2009. p. 163-99.

15. Milczarski P, Bolibok-Bragoszewska H, Myśków B, Stojałowski S, HellerUszyńska K, Góralska M, et al. A high density consensus map of Rye (Secale cereale L.) based on dart markers. PLoS One. 2011;6(12). https://doi.org/10. 1371/journal.pone.0028495.

16. Myśków B, Stojalowski S, Łań A, Bolibok-Bragoszewska H, RakoczyTrojanowska M, Kilian A. Detection of the quantitative trait loci for aamylase activity on a high-density genetic map of rye and comparison of their localization to loci controlling preharvest sprouting and earliness. Mol Breed. 2012;30(1):367-76.

17. Myśków B, Hanek M, Banek-Tabor A, Maciorowski R, Stojałowski S. The application of high-density genetic maps of rye for the detection of QTLS controlling morphological traits. J Appl Genet. 2014:55(1):15-26.

18. Myśków B, Czyczyło-Mysza I, Sokołowska S, Stojałowski S. QTL analysis for moderate drought response in the rye recombinant inbred lines' (RIL) population shows co-localization of QTLs for morphological and physiological traits. ABCbot 2018. in press

19. Gawroński P, Pawełkowicz M, Tofil K, Uszyński G, Sharifova S, Ahluwalia S, et al. DArT markers effectively target gene space in the rye genome. Front Plant Sci. 2016:07(October):1-13. Available from: http://journal.frontiersin. org/article/10.3389/fpls.2016.01600/full

20. Hu J, Zhu L, Zeng D, Gao Z, Guo L, Fang Y, Zhang G, Dong G, Yan M, Liu JQQ. Identification and characterization of NARROW AND ROLLED LEAF 1, a novel gene regulating LEAF morphology and plant architecture in rice. Plant Mol Biol. 2010;73(3):283-92.

21. Masojć $P$, Milczarski $P$, Kruszona P. Comparative analysis of genetic architectures for nine developmental traits of rye. J Appl Genet. 2017;58(3):297-305.

22. Price $A H$, Young EM, Tomos AD. Quantitative trait loci associated with stomatal conductance, leaf rolling and heading date mapped in upland rice (Oryza sativa). New Phytol. 1997;137(1):83-91.

23. Li C, Zou X, Zhang C, Shao Q, Liu J, Liu B, et al. OsLBD3-7 overexpression induced adaxially rolled leaves in rice. PLoS One. 2016;11(6):1-13.

24. Tanaka H, Onouchi H, Kondo M, Hara-Nishimura I, Nishimura M, Machida C, et al. A subtilisin-like serine protease is required for epidermal surface formation in Arabidopsis embryos and juvenile plants. Development. 2001:128(23):4681-9.

25. Parker G. Isolation of COV1, a gene involved in the regulation of vascular patterning in the stem of Arabidopsis. Development. 2003;130(10):2139-48. Available from: http://dev.biologists.org/cgi/doi/10.1242/dev.00441

26. Rawat N, Pumphrey MO, Liu S, Zhang X, Tiwari VK, Ando K, et al. Wheat Fhb1 encodes a chimeric lectin with agglutinin domains and a poreforming toxin-like domain conferring resistance to Fusarium head blight. Nat Genet. 2016:48:1576. Available from: https://doi.org/10.1038/ng.3706

27. Zhang G-H, Xu Q, Zhu X-D, Qian Q, Xue H-W. SHALLOT-LIKE1 is a KANADI transcription factor that modulates Rice leaf rolling by regulating leaf Abaxial cell development. Plant Cell Online. 2009;21(3):719-35. Available from: http://www.plantcell.org/cgi/doi/10.1105/tpc.108.061457

28. Zou LP, Sun XH, Zhang ZG, Liu P, Wu JX, Tian CJ, et al. Leaf Rolling Controlled by the Homeodomain Leucine Zipper Class IV Gene Roc5 in Rice. Plant Physiol. 2011;156(3):1589-602. Available from: http://www. plantphysiol.org/cgi/doi/10.1104/pp.111.176016

29. Li L, Li L, Shi ZY, Shen GZ, Wang XQ, An LS, et al. Overexpression of ACL1 (abaxially curled leaf 1) increased bulliform cells and induced abaxial curling of leaf blades in rice. Mol Plant. 2010:3(5):807-17. Available from: https://doi. org/10.1093/mp/ssq022

30. Lai C-P, Lee C-L, Chen P-H, Wu S-H, Yang C-C, Shaw J-F. Molecular analyses of the Arabidopsis TUBBY-like protein gene family. Plant Physiol. 2004;134(4): 1586-97. Available from: https://www.ncbi.nlm.nih.gov/pmc/articles/ PMC419833/pdf/pp1341586.pdf

31. Liu Q. Identification of rice TUBBY-like genes and their evolution. FEBS J. 2008:275(1):163-71.

32. Yang Z, Zhou Y, Wang X, Gu S, Yu J, Liang G, et al. Genomewide comparative phylogenetic and molecular evolutionary analysis of tubby-like protein family in Arabidopsis, rice, and poplar. Genomics. 2008;92(4):246-53.

33. Hong MJ, Kim DY, Seo YW. Interactions between wheat Tubby-like and SKP1-like proteins. Genes Genet Syst. 2015;90(5):293-304.

34. Ruegger M, Dewey E, Gray WM, Hobbie L, Turner J, Estelle M. The TIR I protein of Arabidopsis functions in auxin response and is related to human SKP2 and yeast Grr1p. Genes Dev. 1998;12(2):198-207. Available from: http:// www.genesdev.org/cgi/doi/10.1101/gad.12.2.198 